\title{
Measurement Techniques for Transmit Source Clock Jitter for Weak Serial RF Links
}

\author{
Chatwin A. Lansdowne Member, IEEE, and Adam M. Schlesinger
}

\begin{abstract}
Techniques for filtering clock jitter measurements are developed, in the context of controlling data modulation jitter on an RF carrier to accommodate low signal-to-noise ratio thresholds of high-performance error correction codes. Measurement artifacts from sampling are considered, and a tutorial on interpretation of direct readings is included.
\end{abstract}

Index Terms-Jitter, Block codes, Phase locked loops

\section{INTRODUCTION}

$\mathrm{N}$ ASA is currently modernizing its communication infrastructure to accompany the development of a Crew Exploration Vehicle (CEV) to replace the Space Shuttle. With this effort comes the opportunity to infuse more advanced coded modulation techniques, including low-density parity check (LDPC) codes that offer greater coding gains than the current capability. The $E_{b} / N_{0}$ thresholds supported by these codes are approaching the Shannon Limit, and because of parity overhead the receiver's symbol synchronizer must function in an even lower $E_{s} / N_{0}$ environment. [1,2,3]

To open up a solution-space for the symbol synchronizer (including at the minimum a tighter loop filter), certain characteristics of transmit data modulation jitter must be controlled [4]. These include rapid phase jitter and slow cycle-to-cycle jitter, which cause symbol error rate degradation in the untracked and tracked frequency bands, respectively. Untracked phase jitter is the accumulation of symbol clock frequency error, and it gains significance as the loop filter is tightened. The loop becomes slower to respond, and phase error has longer to accumulate. Cycle-to-cycle jitter $(\mathrm{CCJ})$ is the rate at which the symbol clock frequency is changing, and as the clock recovery loop tracks out frequency error it must keep pace with this rate of change or a phase error will result.

Jitter measurements are presently made by capturing many contiguous samples of a clock or data signal, then post-

Manuscript received March 1, 2010. Work was performed in the Electronic Systems Test Laboratory (ESTL) in NASA Johnson Space Center's Avionics Systems Division to support the Constellation Program.

Chatwin A. Lansdowne is with the National Aeronautics and Space Administration, Houston, TX 77058 USA (phone: 281-483-1265; fax: 281483-6297; e-mail: chatwin.lansdowne@ nasa.gov).

Adam M. Schlesinger is with the National Aeronautics and Space Administration, Houston, TX 77058 USA (phone: 281-483-0342; fax: 281483-6297; e-mail: adam.m.schlesinger@ nasa.gov). analyzing the clock or data transitions in the capture. Typically an oscilloscope is used to examine a baseband signal. The modulation signal processing can add jitter, so examination of the modulated RF or IF signal as a test point is preferred; no turn-key products exist at this writing, but platforms do exist.

As the second derivative of phase jitter, measurements of cycle-to-cycle jitter are usually dominated by irrelevant (untracked) high-frequency content. Cycle-to-cycle jitter is the product of frequency jitter and jitter rate, so at very low jitter rates the frequency jitter bumps into the tracking range of the clock-recovery loop. It becomes unnecessary to measure cycle-to-cycle jitter below this cut-off rate, which is beneficial because low-rate measurements challenge the memory and processing limits of modern commercially-available instrumentation.

Before discussing measurement accuracy and interpretation, models for two useful filtering techniques are introduced in this paper. These are the Capture Interval Filter, and the NCycle (Large $N$ ) Filter.

\section{CAPTURE INTERVAL FILTERING}

If the capture interval setting of a signal-capture device (e.g. an oscilloscope, signal analyzer, or real-time spectrum analyzer) is much shorter than the period of the phenomenon of interest, then only a fraction of the period will be captured. The average and the root-mean-square (rms) variation from the average depend on location in the cycle, but the rms variation is also attenuated because only a fraction of the total variation was captured and analyzed. This effect of having too short a capture time interval can be exploited as a high-pass measurement filter.

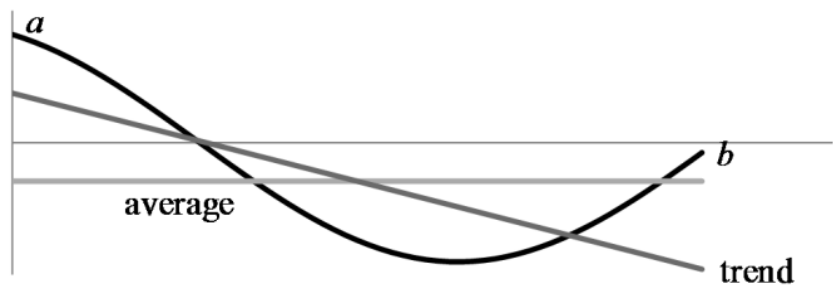

Figure 1. Example Capture of a Fractional Cycle of Tone Jitter

A second relevant approach is to determine rms variation from the linear trend. This has been observed in phase jitter measurements, where at least one vendor determines a reference phase against a clock that is not just the measured 
average rate but can be speeding up or slowing down during the capture according to the measured trend. The result is even faster attenuation of low frequency jitter.

\section{A. RMS from Average}

The jitter packages examined determine rms from average for cycle-to-cycle jitter (CCJ).

If a sinusoid with rms amplitude of one is captured beginning at arbitrary fractional phase $a$ and ending at arbitrary fractional phase $b$ for a duration of $C=b-a$ periods, then the average $A$ of the capture will be

$$
\begin{aligned}
A(a, b) & =\frac{1}{b-a} \int_{a}^{b} \sqrt{2} \sin 2 \pi f t d t \\
& =\frac{\sqrt{2} \cos 2 \pi f a-\cos 2 \pi f b}{2 \pi f(b-a)}
\end{aligned}
$$

The rms, with the average removed will be

$$
r m s(a, b)=\sqrt{\frac{1}{b-a} \int_{a}^{b}(\sqrt{2} \sin 2 \pi f t-A)^{2} d t}
$$

This can easily be expanded and integrated, and then (1) can be substituted to produce a closed-form solution.

$$
\begin{aligned}
& r m s(a, b)=\sqrt{\frac{2}{b-a} \int_{a}^{b}\left(\begin{array}{l}
\left(\frac{1}{2}+\cos 4 \pi f t\right) \\
-2 A \sin 2 \pi f t \\
+A^{2} d t
\end{array}\right)}= \\
& \sqrt{\frac{2}{b-a}\left(\begin{array}{l}
\left(\frac{1}{2}+A^{2}\right)(b-a) \\
+\frac{\sin 4 \pi f b-\sin 4 \pi f a}{4 \pi f} \\
\left.+\frac{2 A(\cos 2 \pi f b-\cos 2 \pi f a}{2 \pi f}\right)
\end{array}\right)}
\end{aligned}
$$

For a single capture taken at an arbitrary time (phase offset) there will be variation in the result. Over several captures this will average out. Therefore it is further necessary to determine the "average" case by evaluating this rms over all possible phases,

$$
\overline{r m s}=\sqrt{\frac{1}{2 \pi} \int_{0}^{2 \pi} r m s^{2}(t, t+C) d t}
$$

Although a closed-form result can be further developed, it does not simplify well and so a numerical approach using three subroutines (the average, the rms, and the average of the rms stepped through all phases) is taken here.

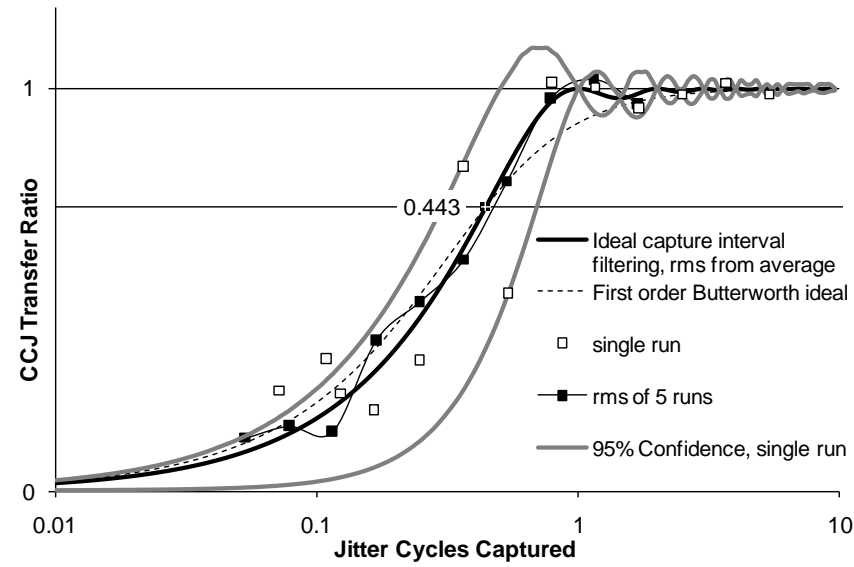

Figure 2. Capture Interval Filter, RMS from Average

This filter effect is shown in Figure 2. It is equivalent to a single-pole filter, with $3 \mathrm{~dB}$ roll-off at 0.443 jitter cycles per capture. The effect was verified using Tektronix DPOJET Plus package, version 2.3; runs were combined by hand as the composite "Std Dev" reported by the software does not remove the averages and so approaches the true rms amplitude of the jitter waveform that has only been captured in segments. The data shows there is variation depending on which part of the waveform was captured, and can be reduced by combining several readings.

\section{B. RMS from Trend}

The Tektronix JIT3 and DPOJET packages determine phase jitter from a reference after removing any linear trend in phase error. This accommodates not only any static phase alignment error but also slowing or hastening trends of the test or sampling oscillators (e.g. due to warm-up) during the measurement.

Trends can be determined by several methods, with leastsquare and bi-square being popular. A numerical approach was used here, and although results shown are using a bisquare fit, the difference from the least-square method is insignificant. The bi-square method is used in practice to reduce sensitivity to outliers that do not occur in this idealized case.

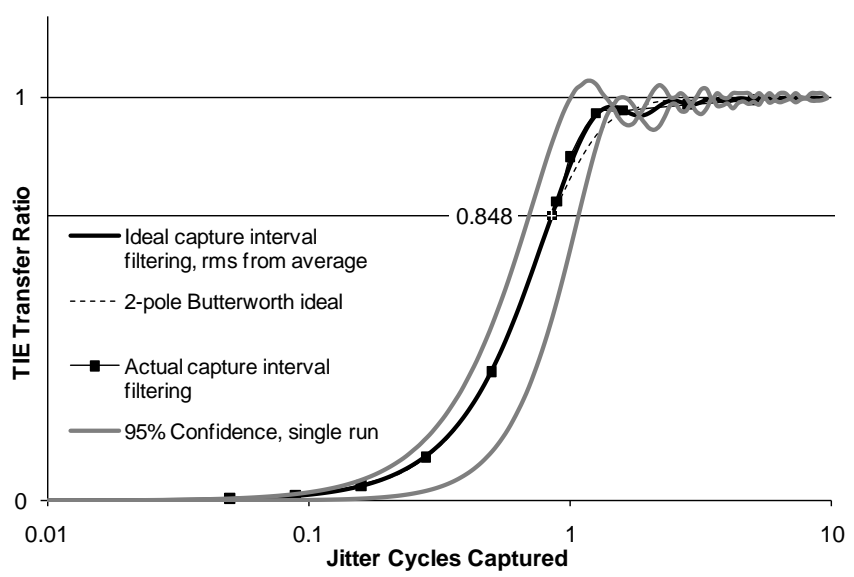

Figure 3. Capture Interval Filter, RMS from Trend 
The filter effect, shown in Figure 3, is equivalent to a secondorder Butterworth filter with $3 \mathrm{~dB}$ roll-off at 0.848 jitter cycles per capture. Data is shown for comparison using the Tektronix JIT3 package. Multiple runs were automatically combined in the software, simply allowing the measurement to settle.

\section{Stabilizing the Measurement}

Finally, the question of the number of readings to be averaged must be addressed. Any analysis of confidence intervals would require assumptions about the distribution of jitter frequencies being measured. Therefore the most practical statement to make is that if measurements appear to lack repeatability due to long-term wander then the technique described here should be used to combine multiple measurements until a suitably stable reading is attained.

\section{FILTERING By N-CyCle MEASUREMENTS}

Rather than measure Cycle-to-Cycle jitter between adjacent cycles, an $\mathrm{N}$-cycle measurement with large $N$ can be used as a low-pass filter. The theory behind this filter is conceptually similar to that for the capture interval filter. In this case, one examines difference between the collective duration of the trailing $\mathrm{N}$ cycles by comparison with the following $\mathrm{N}$ cycles. The $2 \mathrm{~N}$ measurement window can slide by a cycle at a time.

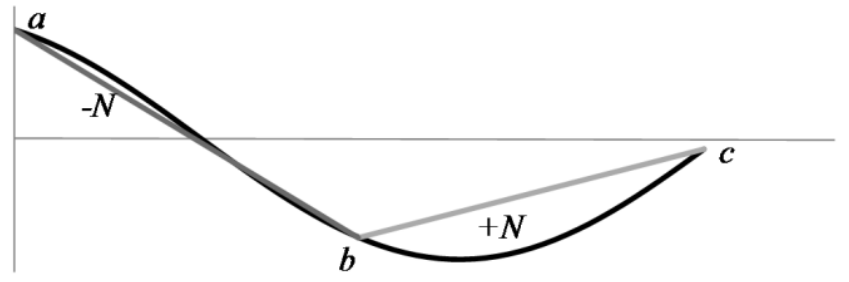

Figure 4. Example N-cycle Measurement for Tone Jitter

If $a \approx b-N / f_{c}$ and $c \approx b+N / f_{c}$, then the N-cycle jitter is the difference between these durations of the $N$ cycles before $b$ and the $N$ cycles after $b$. For a tone with rms amplitude of one and normalized to change during $\mathrm{N}$ periods per $\mathrm{N}$ periods,

$$
N C J=\left(\frac{f_{c}}{N}\right)^{2}\left(\begin{array}{r}
\int_{a}^{b} \int \sqrt{2} \cdot \cos \left(2 \pi f_{m} t\right) d^{2} t \\
-\int_{b}^{c} \int \sqrt{2} \cdot \cos \left(2 \pi f_{m} t\right) d^{2} t
\end{array}\right)
$$

and then the rms measurement can be found from

$$
r m s_{N C J}=\sqrt{\frac{1}{2 \pi} \int_{0}^{2 \pi} N C J^{2}(\Phi) d \Phi}
$$

where $\Phi=2 \pi f_{m} b$. The integration can be simplified to produce a closed form measurement filter transfer function,

$$
r m s_{N C J}=-\frac{1}{2 \pi^{2}} \cdot\left(\frac{f_{c}}{N f_{m}}\right)^{2} \cdot\left(\cos \left(\frac{2 \pi N f_{m}}{f_{c}}\right)-1\right)
$$

The filter effect, shown in Figure 5, is equivalent at $N=1000$ to a second-order Butterworth filter at $1000 f_{m} / f_{c}=0.32$ cycles. For comparison, data is shown that was taken quickly using the Agilent EZJIT package with an Infinium DSO9404A platform.

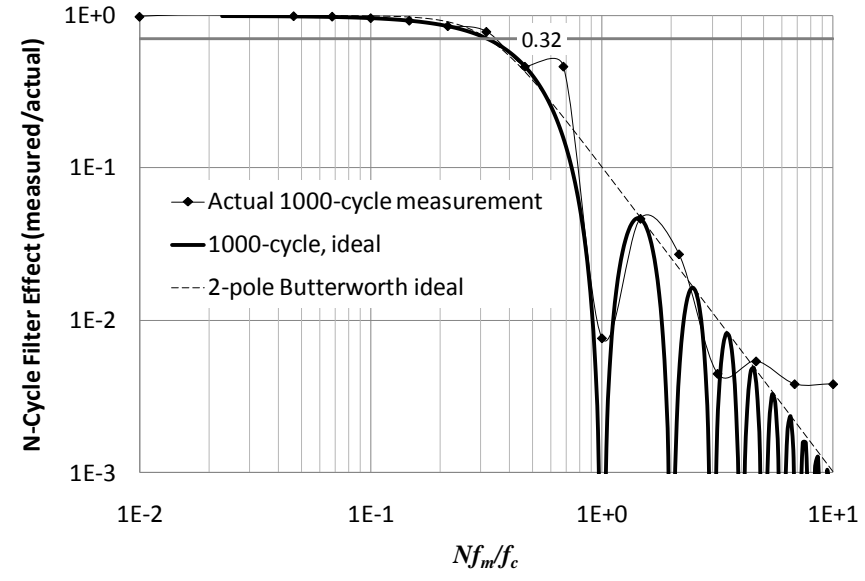

Figure 5. 1000-Cycle Filter for Cycle-to-Cycle Jitter

The N-cycle measurement is elegant because it scales automatically with data rate, so it is easy to specify and easy to implement. N-cycle measurements also have the property of magnifying the jitter measurement by $N^{2}$. This means that a CCJ specification limit can be expressed as a simple percentage instead of more alarming and awkward scientific or engineering notation (e.g. " $1 \% 1000$-cycle jitter" instead of "1E-8 CCJ"). The drawback of this technique is the obvious blind spots which presumably would be placed near the transition region of a receiver's clock recovery loop.

Averaging and confidence interval for the N-Cycle measurement are not addressed because the technique is a low-pass filter and adequate averaging should occur during the capture so that the measurement is repeatable.

\section{JitTER MEASUREMENT SAMPLING ARTIFACT}

When transition times are determined from post-processing a list of samples of an ideal square waveform, the actual transition time can only be known to fall somewhere between a pair of samples. For example, rising edge jitter measurement error is illustrated in Figure 6. Modern oscilloscopes can be purchased with a large amount of highspeed capture memory and can post-process quickly but the user will always need to trade between samples per cycle and number of clock cycles in the capture when measuring small amounts of slow jitter.

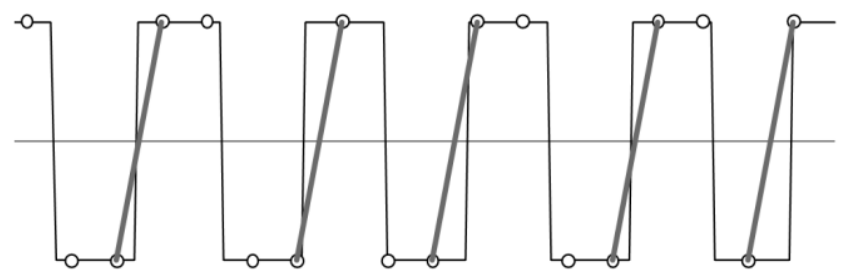

Figure 6. Measurement Bias Artifact Caused by Sampling

From Figure 6, if rising edges are determined by straightline interpolation then the mid-point will be declared the transition time and the actual transition time could differ by $\pm T_{s} / 2$ (peak phase error). If both clocks are actually ideal, then the error cycles as a saw-tooth waveform with a beat-note frequency of $f_{\text {beat }}=f_{c}\left(f_{s} / f_{c}-\left[f_{s} / f_{c}\right]\right)$ where the square brackets denote rounding to the nearest integer. The sawtooth 
waveform has a crest factor of $\sqrt{3}$, meaning that the artifact contributes an expected rms phase error of $T_{s} / \sqrt{12}$ to the measurement. The phase ramp in turn appears as a bias in the length of the clock periods, punctuated by sharp corrections at the beat frequency. The apparent cycle-to-cycle change in the length of each clock cycle is negligible, except for disturbances at the beat frequency.
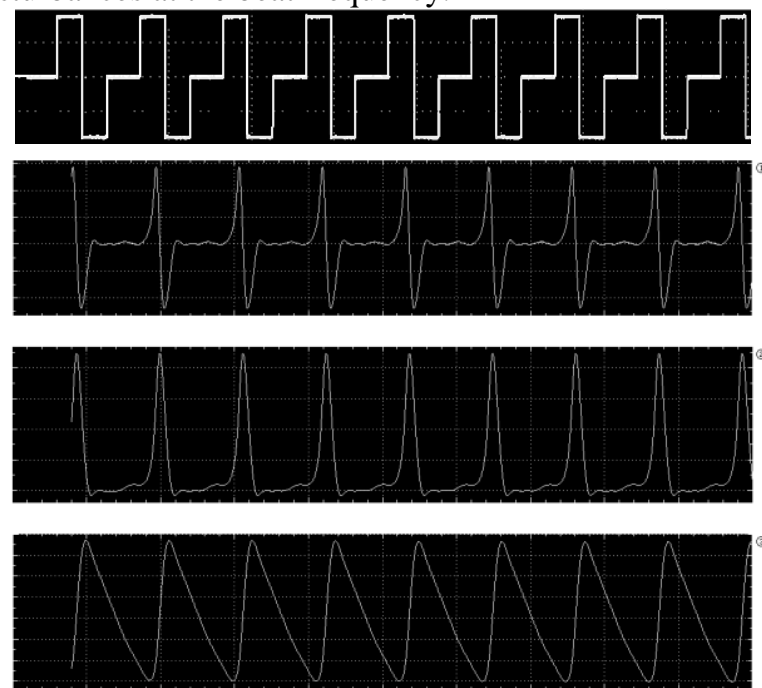

Figure 7. Sampling Artifact in NCJ, CCJ, PJ, and TIE

This leaves us with the question, how much frequency jitter and cycle-to-cycle jitter artifact do we expect. First, we need a mathematical description of these waveforms. Begin with phase jitter normalized to $f_{c}$ and with peak-to-peak amplitude of $T_{s}$, and then differentiate in two stages.

$$
\begin{aligned}
& \Phi_{e}(t)=T_{s} f_{c}\left(f_{\text {beat }} t-\left\lfloor f_{\text {beat }} t\right\rfloor-1 / 2\right) \\
& f_{e}(t)=\frac{d \Phi_{e}(t)}{d t}=T_{s} f_{c}\left(T_{s} f_{\text {beat }}-T_{s} \delta\left(n / f_{\text {beat }}\right)\right) \\
& f_{e}{ }^{\prime}(t)=T_{s}^{2} f_{c} \delta^{\prime}\left(n / f_{\text {beat }}\right)
\end{aligned}
$$

where $n$ is any integer.

But the $\delta\left(n / f_{\text {beat }}\right)$ function is observed in a discrete-time measurement as a cyclic train of $f_{c} / f_{\text {beat }}-1$ cycles that are each $T_{s} /\left(f_{c} / f_{\text {beat }}-1\right)$ short or long, followed by a "pulse" of one clock cycle with one extra or missing sample period. Thus the measured normalized peak-to-peak period jitter is $T_{s} f_{c}$ and the rms period jitter is

$$
\begin{aligned}
P J_{r m s, \text { norm }} & =\sqrt{\frac{f_{\text {beat }}}{f_{c}}\left(\left(\frac{f_{c}}{f_{\text {beat }}}-1\right)\left(\frac{f_{\text {beat }}}{f_{c}}\right)^{2}+\left(\frac{f_{\text {beat }}}{f_{c}}-1\right)^{2}\right)} \\
& =T_{s} \sqrt{f_{\text {beat }} f_{c} /\left(1-f_{\text {beat }} / f_{c}\right)} \\
& \approx T_{s} \sqrt{f_{\text {beat }} f_{c}}
\end{aligned}
$$

Further analysis using the Fourier series would be possible. Similarly, the peak-to-peak cycle-to-cycle jitter is $2 T_{s} f_{c}$ and the rms cycle-to-cycle jitter is

$$
\begin{aligned}
C C J_{r m s, \text { norm }} & =\sqrt{\frac{f_{\text {beat }}}{f_{c}}\left(0^{2}+2\left(T_{s} f_{c}\right)^{2}\right)} \\
& =T_{s} \sqrt{2 f_{\text {beat }} f_{c}}
\end{aligned}
$$

In practice, several factors attenuate the artifact below these expected values; these include jitter measurement filters, risetime and ringing of the clock or data pulses, and configuration of any anti-aliasing pre-filter. The oscilloscope was observed to outperform the model, most significantly when the rise and fall times are a large fraction of a clock cycle or sample period.
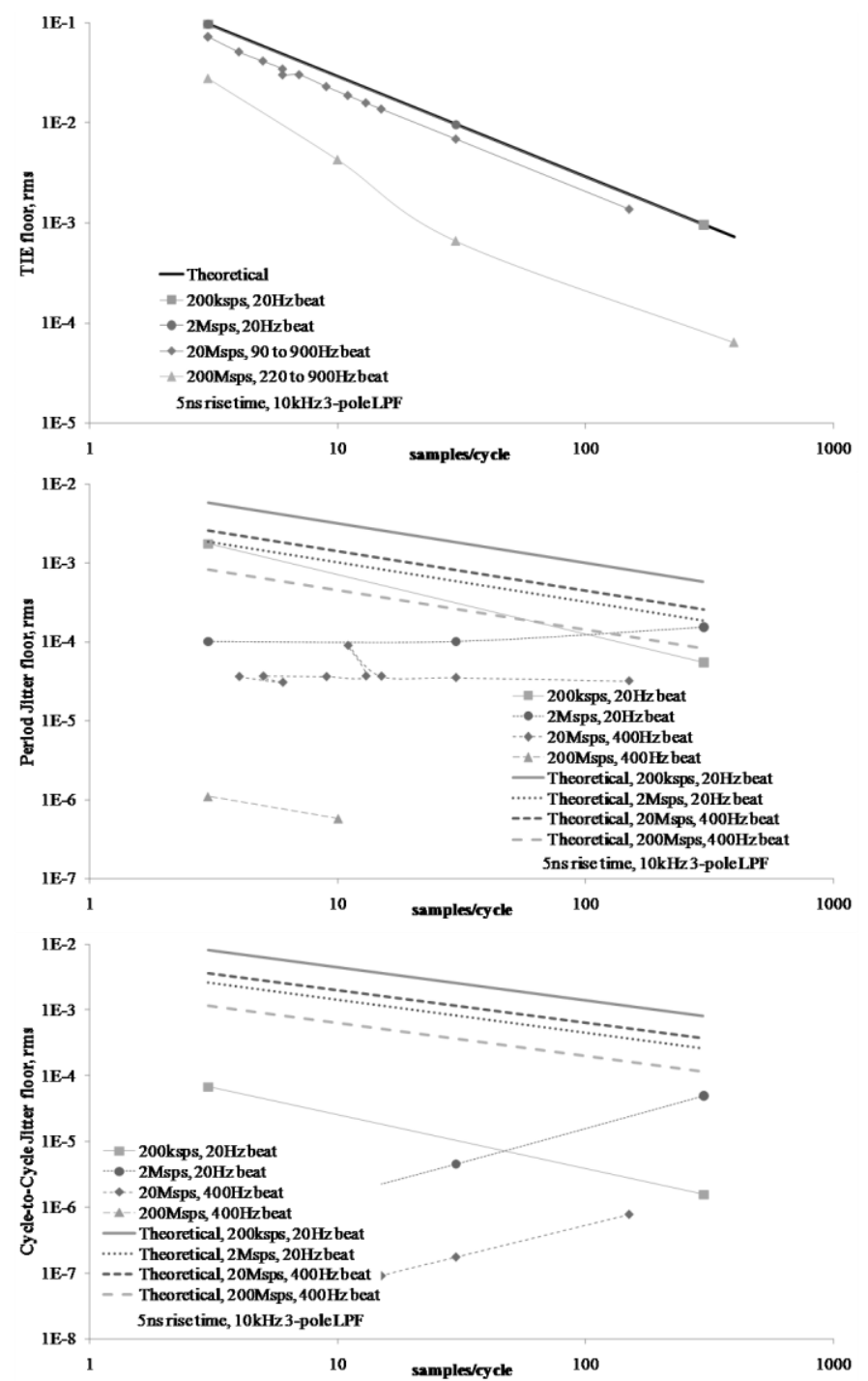

Figure 8. Measured and Expected Sampling Artifact

In conclusion, the sample rate can beat against the data rate at beat-note frequencies that cannot be filtered; this is aggravated by typical even-multiple-of-ten sample rates examining even-multiple-of-ten data rates. The result appears as phase jitter, frequency offset, and peak-to-peak period and cycle-to-cycle jitter. Faster sampling helps, but even with hundreds of samples per cycle a slow beat can be significant. However, while these waveshapes can have an intimidating peak-to-peak their contribution to the rms measurements recommended in [4] is much smaller. 
The beat-note is identifiable because it changes with settings. It can be filtered in the jitter package if it can be moved out of the frequency band of interest. For example, a faster beat-note increases the rms CCJ and rms FJ floors, except that a faster beat note is at some point reclassified as phase jitter.

\section{RECONCILING MEASUREMENTS AND NORMALIZING TO DATA RATE}

\section{A. Relating Period and Frequency Jitter}

Noise on the control voltage for a voltage-controlled oscillator creates frequency jitter through the process of frequency modulation for which good models exist. But the frequency of a clock can only be directly measured by sampling once per period; that is, frequency jitter is a continuous-time phenomenon that can only be observed as discrete-time period jitter. The two terms are practically interchangeable, but there is a subtle difference. Given an average clock frequency $f_{c}$ with corresponding period $T_{c}=1 / f_{c}$, and an error frequency $\Delta f$. The normalized frequency jitter is $F J=\Delta f / f_{c}$, the frequency displacement range is $f_{c} \pm \Delta f$, and the period errors are $T_{\max }=1 /\left(f_{c}-\Delta f\right)$ and $T_{\min }=1 /\left(f_{c}+\Delta f\right)$. From this it can be shown that the normalized period errors are,

$$
\begin{aligned}
& \frac{T_{\max }-T_{c}}{T_{c}}=\frac{\Delta f / f_{c}}{1-\Delta f / f_{c}} \\
& \text { and } \frac{T_{c}-T_{\min }}{T_{c}}=\frac{\Delta f / f_{c}}{1+\Delta f / f_{c}}
\end{aligned}
$$

The point is that the terms "frequency" and "period" jitter are interchangeable only when $\Delta f<f_{c}$ and the expressions show that the period jitter is more by a factor of the normalized frequency jitter; that is, frequency jitter of $1 \%$ is actually equivalent to period jitter of $1.01 \%$.

\section{B. Tutorial for Normalizing and Interpreting Measurements}

Phase jitter, frequency jitter, and cycle-to-cycle jitter dimensions can all be normalized to the data rate. Instruments generally report measurements in absolute time units which then need to be converted; it can be difficult to keep track of the arithmetic in one's head. For the benefit of someone just learning to use one of the available jitter measurement packages, let us consider the example case of a $1 \mathrm{MHz}$ squarewave frequency modulated $\pm 1 \mathrm{kHz}$ peak (" $0.1 \%$ peak") at a single frequency of $1 \mathrm{kHz}$. By definition, this is $0.1 \%$ peak frequency jitter at a $0.1 \%$ rate. The jitter package can only directly observe period jitter: it will report that the periods are $1 \mu$ s on average but range between 1.001 us and 0.999 us with a standard deviation of $714 \mathrm{ps}$. Multiplying by the known crest factor $\sqrt{2}$ for the tone case would give a period jitter of $1.001 \%$ peak, or a frequency jitter of $1 \%$ peak.

Then we expect about $15.9 \%$ peak phase jitter, equivalent to $11.25 \%$ rms. An instrument would report $112.5 \mathrm{~ns} \mathrm{rms}$, which relative to the $1 \mu \mathrm{s}$ period also reported by the same instrument matches the expected phase error.
Finally, the cycle-to-cycle jitter would be reported by the package as $4.43 \mathrm{ps} \mathrm{rms/cycle.} \mathrm{This} \mathrm{gives} \mathrm{a} \mathrm{result} \mathrm{of}$ $4.43 \cdot 10^{-6} /$ cycle normalized to the data rate. The tone jitter equation anticipates the amplitude of $4.43 \mathrm{MHz} / \mathrm{sec} \mathrm{rms}$.

Observe that phase jitter is normalized to the clock period, frequency jitter is normalized to the data rate, and cycle-tocycle jitter is normalized to the square of the data rate.

\section{ACKNOWLEDGMENT}

The authors offer thanks to B. G. Smith for his technical mentorship and for challenges to our explanations. The authors also appreciate the technical exchanges arranged through Charles Conger of Tektronix and the loan of equipment arranged through Thomas McNulty of Agilent Technologies.

\section{REFERENCES}

[1] K. S. Andrews, D. Divsalar, S. Dolinar, J. Hamkins, C. R. Jones, F. Pollara, "The Development of Turbo and LDPC Codes for Deep Space Applications," Proceedings of the IEEE, Vol. 95, No. 11, November 2007.

[2] "Low Density Parity Check Codes For Use In Near-Earth and Deep Space Applications," CCSDS 131.1-O-2, Consultative Committee for Space Data Systems, September 2007

[3] "TM Synchronization and Channel Coding-Summary of Concept and Rationale," CCSDS 130.1-G-1, Consultative Committee for Space Data Systems, June 2006

[4] C. A. Lansdowne, A. M. Schlesinger, M. K. Cheng, D. K. Lee, "JitterInduced Symbol Slip Rates in Next-Generation Ground Segment Receivers,” AIAA SpaceOps 2010, Huntsville AL USA, April 25-30, 2010 